\title{
Preclinical Efficacy and Clinical Feasibility of a Novel Aerosol- Exposure Protection Mask for Esophagogastroduodenoscopy
}

\author{
Mai Ego Makiguchi', Seiichiro Abe', Yutaka Okagawa ${ }^{1,2}$, Satoru Nonaka', Haruhisa Suzuki', Shigetaka Yoshinaga', Ichiro Oda', \\ Okamoto Ryuta ${ }^{3}$, and Yutaka Saito ${ }^{1}$
}

${ }^{1}$ Endoscopy Division, National Cancer Center Hospital, Tokyo, ${ }^{2}$ Department of Gastroenterology, Tonan Hospital, Sapporo, Hokkaido, ${ }^{3}$ Shin Nippon Air Technologies, Tokyo, Japan

Background/Aims: This study aimed to assess the efficacy of a novel aerosol-exposure protection (AP) mask in preventing coronavirus disease in healthcare professionals during upper gastrointestinal endoscopy and to evaluate its clinical feasibility. Methods: In Study 1, three healthy volunteers volitionally coughed with and without the AP mask in a cleanroom. Microparticles were visualized and counted with a specific measurement system and compared with and without the AP mask. In Study 2 , 30 patients underwent endoscopic resection with the AP mask covering the face, and the SpO2 was measured throughout the procedure.

Results: In Study 1, the median number of microparticles in volunteers 1, 2, and 3 with and without the AP mask was 8.5 and 110.0 , 7.0 and 51.5 , and 8.0 and 95.0 , respectively $(p<0.01)$. Using the AP mask, microparticles were reduced by approximately $92 \%$. The median distances of microparticle scattering without the AP mask were 60, 0, and 68 in volunteers 1, 2, and 3, respectively. In Study 2 , the mean $\mathrm{SpO} 2$ was $96.3 \%$, and desaturation occurred in three patients.

Conclusion: The AP mask could provide protection from aerosol exposure and can be safely used for endoscopy in clinical practice. Clin Endosc 2022;55:226-233

Key Words: COVID-19; Gastrointestinal endoscopy; Personal protective equipment

\section{INTRODUCTION}

The new coronavirus, severe acute respiratory syndrome coronavirus 2 (SARS-CoV-2), rapidly spread throughout the world, with over 83 million patients becoming infected with it and over 1.8 million deaths globally by January $5,2021{ }^{1}$ According to previous reports, the routes of SARS-CoV-2 transmission include direct contact (i.e., contact with respiratory droplets and aerosols from an affected person) and indirect

Received: June 1,2021 Revised: August 4, 2021

Accepted: August 9, 2021

Correspondence: Seiichiro Abe

Endoscopy Division, National Cancer Center Hospital, 5-1-1 Tsukiji, Chuo-ku, Tokyo 104-0045, Japan

Tel: +81-3-3542-2511, Fax: +81-3-3542-3815, E-mail: seabe@ncc.go.jp

ORCID: https://orcid.org/0000-0002-2736-6921

(c) This is an Open Access article distributed under the terms of the Creative Commons Attribution Non-Commercial License (http://creativecommons.org/ licenses/by-nc/3.0) which permits unrestricted non-commercial use, distribution, and reproduction in any medium, provided the original work is properly cited. contact, such as contact with contaminated surfaces or supplies. $^{2}$

Healthcare professionals (HCPs) are particularly at risk of infection. It has been reported that approximately $20 \%$ of the HCPs in Italy have been infected with coronavirus during the pandemic. ${ }^{3}$ In addition, HCPs involved in the endoscopy unit are at an increased risk of SARS-CoV-2 infection from inhalation of airborne droplets, conjunctival contact, and potential fecal-oral transmission. ${ }^{4}$ Sagami et al. measured $0.3-1.0 \mu \mathrm{m}$ aerosols during upper gastrointestinal (GI) endoscopy and concluded that esophagogastroduodenoscopy (EGD) was an aerosol-generating procedure. ${ }^{5}$ Worldwide, guidelines have issued recommendations for conducting endoscopic procedures during the coronavirus disease (COVID-19) pandemic., ${ }^{4,-10} \mathrm{To}$ prevent HCPs from contracting COVID-19 during endoscopic procedures, appropriate personal protective equipment (PPE) is needed. International guidelines recommend PPE based on the risk of transmission. ${ }^{4,6-10}$ For patients who are negative for COVID-19 and are not suspected to have COVID-19, HCPs 
should perform endoscopies with PPE, including a face mask, isolation gown with water resistance, and eye protection. For patients with suspected, probable, or confirmed COVID-19, enhanced PPE should be used during endoscopy, including the use of an N95 mask, isolation gown with water resistance, head cover, eye protection, and face shield. ${ }^{6}$

Protection of HCPs is well established as mentioned above; however, protection of HCPs from the patient's side has not yet been standardized. Consequently, we developed a novel aerosol-exposure protection (AP) mask for upper GI endoscopy, with a plastic file folder covering the patients' faces. This study aimed to evaluate the preclinical efficacy of aerosol exposure prevention and the clinical feasibility of AP masks during therapeutic endoscopy.

\section{METHODS}

\section{AP mask}

The AP mask was made of an A4-sized plastic file folder as follows (Fig. 1). i) The corner of a plastic file folder was cut into a square approximately $12.5 \times 12.5 \mathrm{~cm}$ in size. ii) To create space for insertion of the gastroscope, $3.5 \mathrm{~cm}$ of the corner was cut. The diameter of the space created for inserting the EGD was approximately $15 \mathrm{~mm}$, which was the minimum size required to insert a therapeutic endoscope with a transparent cylinder. iii) Both opened sides were covered by two gauze pads, allowing for the absorption of saliva. iv) A hole was punctured, penetrating the gauze pad and the plastic folder, on both corners. v) An elastic string was tied through both holes.

\section{Study design}

First, an exploratory study was performed to investigate the efficacy of aerosol-exposure prevention by the AP mask in a preclinical setting in a clean room where we could eliminate air dust and precisely visualize and measure the aerosol. Study 1 was not conducted in a real clinical setting because of the need for these specific requirements and limited space. Study 1 included only three volunteers. Three healthy volunteers were recruited from the National Cancer Center Hospital, Tokyo, Japan. In Study 2, the AP mask was clinically tested in patients with early esophageal and gastric endoscopic mucosal
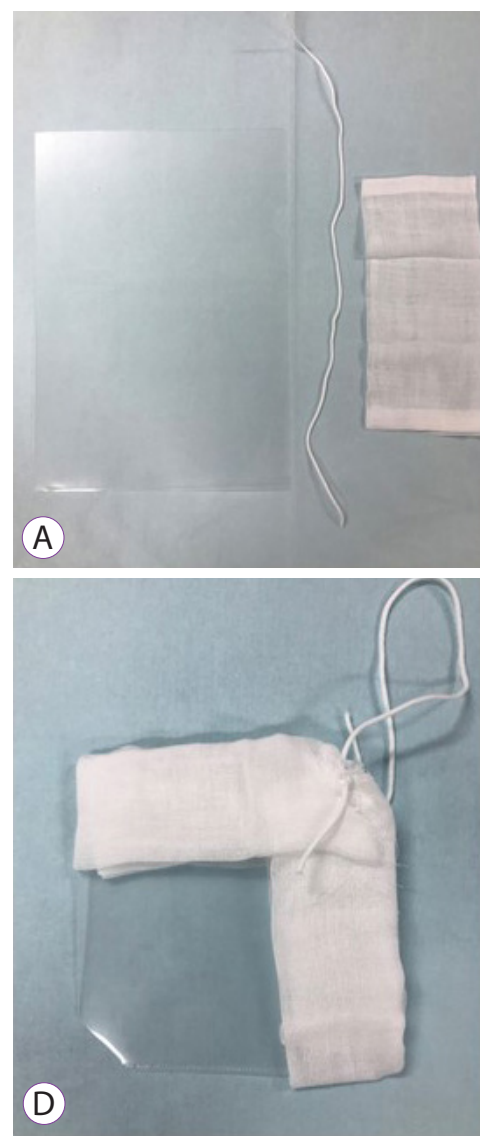
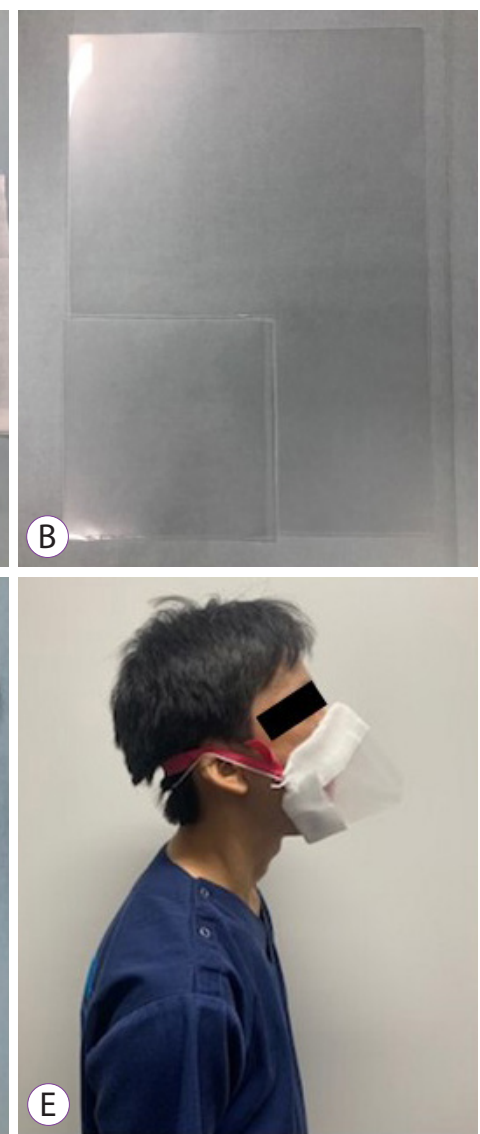

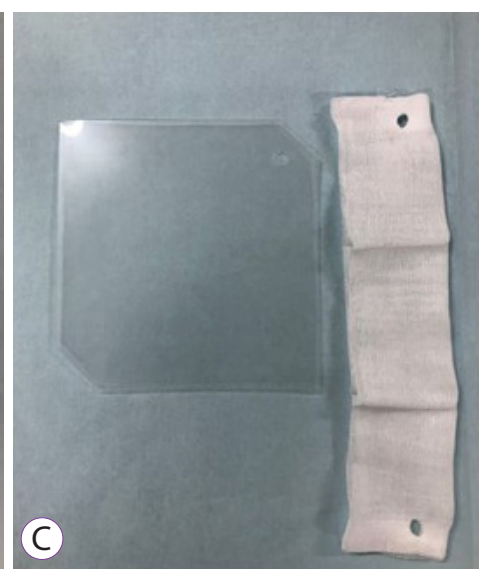

Fig. 1. How to make aerosol-exposure protection (AP) mask. (A) Materials of the AP mask: an A4-sized plastic folder, string, and two pieces of gauze pad. (B) An A4-sized plastic folder was cut into a $12.5 \times 12.5 \mathrm{~cm}$ square, and a $3.5 \mathrm{~cm}$ square was cut from its corner. (C) To insert the string, a small hole was made in the plastic folder and two pieces of gauze pad. (D) The gauze was placed on the edges of the plastic file holder with a string. $(E)$ A healthy volunteer wearing the mask. 
resection (EMR) and endoscopic submucosal dissection (ESD). This study was carried out in accordance with the ethical principles defined by the Declaration of Helsinki and approved by the Institutional Review Board (2020-027) on July 13, 2020, at the National Cancer Center Hospital. Our protocol was registered on June 20, 2020, at the University Hospital Medical Information Network Clinical Trials Registry (UMIN) as UMIN000040815. Written informed consent for this study was obtained from all participants.

\section{Study phases}

\section{Study 1: pre-clinical study}

Three healthy volunteers (Seiichiro Abe, Mai Ego Makiguchi, Yutaka Okagawa) checked that they were afebrile and asymptomatic relating to COVID-19 before the study. A COVID-19 polymerase chain reaction (PCR) test was not performed due to limited resources during the pandemic in Japan. ${ }^{11}$ First, volunteers were laid down on a bed in the left lateral position in a cleanroom. The cleanroom was designed to eliminate dust and maintain the number of microparticles in the air at almost zero. Second, as a preclinical simulation, a standard gastroscope (GIF-H260; Olympus Medical, Tokyo,
Japan) was inserted into the mouth of the volunteer through an endoscopic mouthpiece (Endoleader multi-type Z; Top Corporation, Tokyo, Japan) with and without the AP mask. Third, the volunteers volitionally coughed 12 times at 20-second intervals with and without the AP mask, and the microparticles were visualized during the endoscopy simulation. A short-wavelength visible light source $(400 \mathrm{~nm})$ was illuminated from a light-emitting diode (LED) source (Parallel Eye D; Shin Nippon Air Technologies, Tokyo, Japan) and directed to a video camera (Eye Scope; Shin Nippon Air Technologies, Tokyo, Japan), which was set on the other side of the Parallel Eye (Fig. 2 ). The eye scope captured microparticles in the cleanroom. Video of microparticle visualization, mostly aerosol, were recorded and edited using an image processing system software (Particle Eye viewer; Shin Nippon Air Technologies, Tokyo, Japan) (Fig. 3, Supplementary Videos 1 and 2). Microparticles as small as $0.5 \mu \mathrm{m}$ during the coughs were counted from the video image data with 6 frames per 0.2 second using a portable particle visualization system (Type-S; Shin Nippon Air Technologies, Tokyo, Japan), which allows for real-time visualization and measurement of microparticles. We compared the number of microparticles for 10 seconds with and without the AP mask. In addition, the microparticle scatter distance was
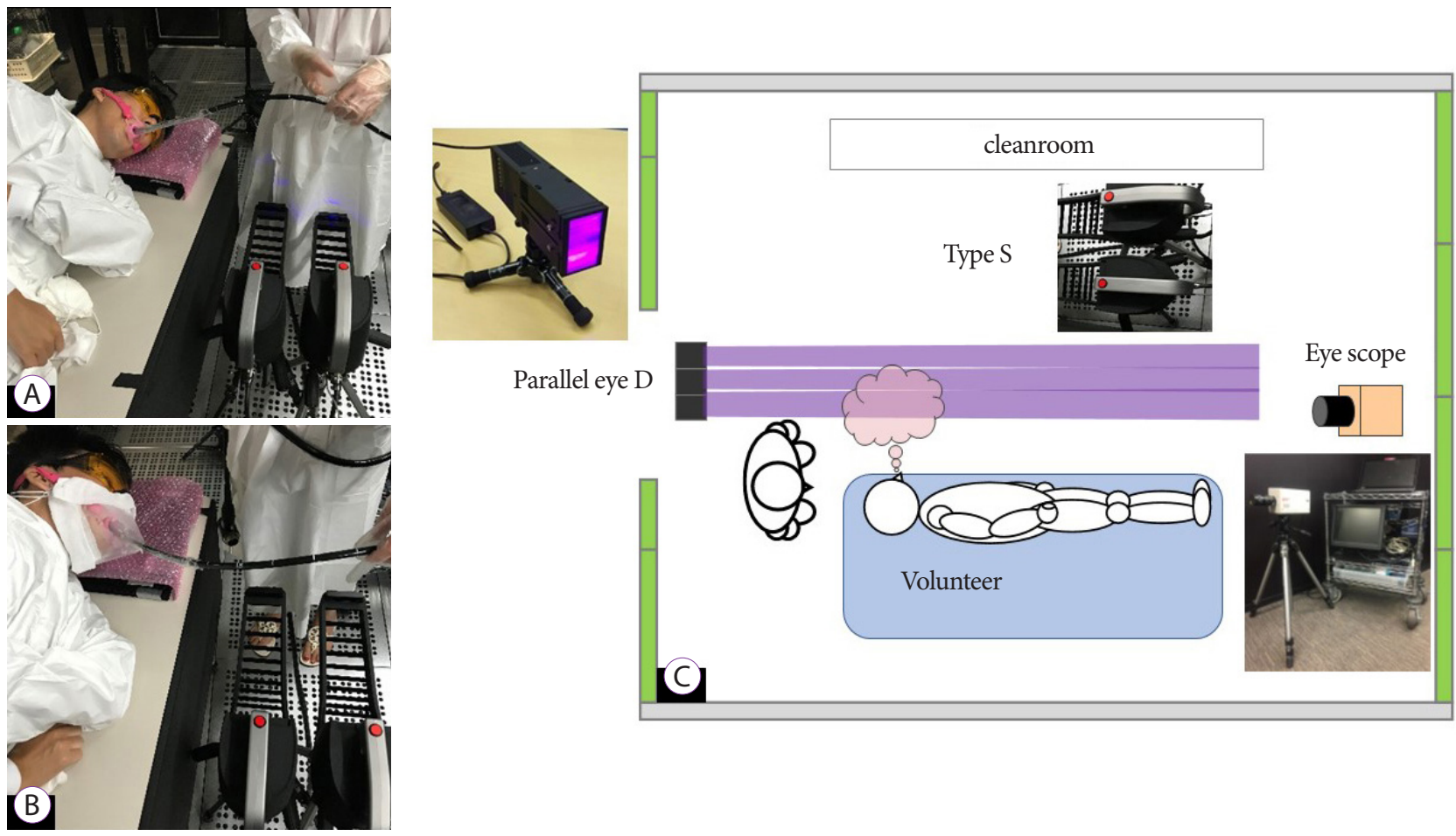

Fig. 2. Schematic illustration of experimental design in a cleanroom. (A) A healthy volunteer without the aerosol-exposure protection (AP) mask. (B) A healthy volunteer with the AP mask. (C) Schematic illustration of the experimental design in a cleanroom. 


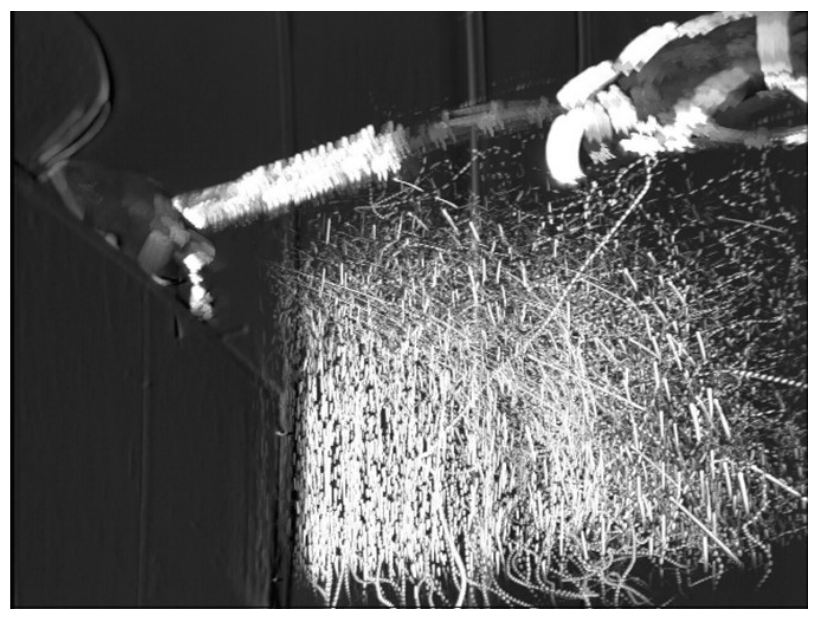

Fig. 3. Visualization of aerosol particles by light-emitting diode during simulated endoscopy.

measured from the videos based on a $0.128 \mathrm{~cm} /$ pixel length.

Study 1 assessed the effectiveness of aerosol exposure protection with and without the AP mask using aerosol particle visualization analysis.

\section{Study 2: clinical use of the AP mask during endoscopic resection}

Study 2 aimed to evaluate the clinical feasibility of the AP mask. Patients undergoing EMR or ESD for early esophageal cancer and early gastric cancer were enrolled from May to July 2020 at the National Cancer Center Hospital.

ESD/EMR was performed following the Japan Gastroenterological Endoscopy Society (JGES) recommendations for GI endoscopy during the COVID-19 pandemic. ${ }^{7}$ The inclusion criteria were as follows: a) patients undergoing endoscopic resection (including ESD and EMR) for superficial esophageal cancer or early gastric cancer, b) age $>20$ years, and c) performance status (PS) $0-1$. The exclusion criteria were as follows: a) positive PCR for COVID-19 and b) patients who were suspicious for infection of COVID-19, namely patients with any respiratory symptoms or fever $\left(>37.5^{\circ} \mathrm{C}\right)$, high-risk exposure to a confirmed COVID-19 contact or travel overseas within two weeks before EMR/ESD, severe fatigue or respiratory symptoms, new loss of taste or smell, and diarrhea lasting at least 4-5 days. All endoscopic treatments were performed with appropriate PPE, following the guidelines described above. ${ }^{4,6-10}$

\section{Endoscopic procedure}

EMR and ESD were performed as described previously.12,13 Briefly, for EMR, saline was injected into the submucosa after marking dots around the lesion. The lesion was suctioned into the cap and resected.

The ESD procedure was performed as follows: first, marking dots were drawn around the lesion; second, saline or hyaluronic acid was injected into the submucosa; and third, mucosal incision and submucosal dissection were made using a needle knife and IT knife 2 (KD-611L; Olympus Medical, Tokyo, Japan). EMR and ESD were generally performed using a therapeutic endoscope for ESD (GIF-Q260J; Olympus Medical, Tokyo, Japan) and a standard endoscope for EMR (GIF-H260; Olympus Medical, Tokyo, Japan) and the following generators: ESG-100 (Olympus Medical, Tokyo, Japan) or VIO 3 (ERBE, Tübingen, Germany).

In our hospital, we performed ESD with propofol-based deep sedation. Propofol was administered using a target-controlled infusion pump with an initial target blood concentration of $2 \mu \mathrm{g} / \mathrm{ml}$; if necessary, the target blood concentration was changed by $0.1-0.2 \mu \mathrm{g} / \mathrm{ml} .{ }^{14}$ In addition, scopolamine butyl bromide was used as an antispasmodic, and pentazocine was used as an analgesic. During endoscopic treatment, the patients received continuous supplemental oxygen at $2 \mathrm{~L} / \mathrm{min}$ using a nasal cannula under the AP mask.

Electrocardiogram, oxygen saturation, and non-invasive blood pressure measurements were constantly monitored during sedation. Desaturation was defined as $\mathrm{SpO} 2<94 \%$.

The endpoints of Study 2 were to evaluate the safety of AP masks used during clinical endoscopic procedures and the presence of adverse events such as oxygen desaturation and pneumonia.

\section{Statistical analysis}

In Study 1, continuous variables are shown as medians and ranges. Continuous data were compared using the Wilcoxon Mann-Whitney $U$ test. All p-values were reported as two-sided, and a $p$-value $<0.05$ was considered statistically significant. In Study 2, continuous variables are shown as mean and standard deviation. Statistical analyses were performed using SPSS (version 23; SPSS Inc., Chicago, IL, USA).

\section{RESULTS}

\section{Study 1}

Tables 1 and 2 summarize the number of microparticles generated after coughing five times with and without the AP mask. The median (range) number of microparticles with/ without the AP mask was $8.5(4-79) / 110.0(48-231)(p<0.01)$, $7.0(4-22) / 51.5(26-143)(p<0.01)$, and $8.0(1-20) / 95.0$ $(11-791)(p<0.01)$ in volunteers 1,2 , and 3 , respectively. The 
median (range) number of microparticles with and without the AP mask in the three volunteers was 86.5 (11-791) and 7.0 $(1-79)$, respectively $(p<0.01)$. There was a significant difference between the two groups. Using the AP mask, microparticles were reduced by approximately $92 \%$.

Table 1. The Number of Aerosol Particles With/Without the Aerosol-Exposure Protection Mask

\begin{tabular}{lccc}
\hline & With the mask & Without the mask & $\boldsymbol{p}$-value \\
\hline Volunteer 1 & $8.5(4-79)$ & $110.0(48-231)$ & $<0.01$ \\
Volunteer 2 & $7.0(4-22)$ & $51.5(26-143)$ & $<0.01$ \\
Volunteer 3 & $8.0(1-20)$ & $95(11-791)$ & $<0.01$ \\
\hline
\end{tabular}

Data are presented by median (range).

Table 2. The Number of Aerosol Particles from the Volunteer's Mouth

\begin{tabular}{lccc}
\hline $\begin{array}{l}\text { AP Mask With/ } \\
\text { Without }\end{array}$ & Volunteer 1 & Volunteer 2 & Volunteer 3 \\
\hline 1 & $55 / 113$ & $9 / 84$ & $3 / 39$ \\
\hline 2 & $15 / 74$ & $7 / 143$ & $10 / 11$ \\
\hline 3 & $15 / 107$ & $7 / 57$ & $7 / 791$ \\
\hline 4 & $4 / 48$ & $15 / 31$ & $1 / 78$ \\
\hline 5 & $4 / 99$ & $7 / 30$ & $6 / 65$ \\
\hline 6 & $31 / 48$ & $22 / 131$ & $6 / 158$ \\
\hline 7 & $9 / 64$ & $10 / 26$ & $12 / 45$ \\
\hline 8 & $4 / 231$ & $7 / 43$ & $12 / 45$ \\
\hline 9 & $6 / 226$ & $5 / 44$ & $9 / 227$ \\
\hline 10 & $8 / 115$ & $7 / 89$ & $9 / 112$ \\
11 & $7 / 140$ & $4 / 46$ & $9 / 112$ \\
\hline 12 & $79 / 144$ & $5 / 92$ & $5 / 347$ \\
\hline
\end{tabular}

AP, aerosol-exposure protection.

Table 3. The Distance Generated Aerosol Particles during Cough With and Without the Aerosol-Exposure Protection Mask

\begin{tabular}{lccccc}
\hline Without mask $(\mathrm{cm})$ & & & & & \\
\hline Volunteer 1 & 60 & 60 & 60 & 60 & 60 \\
Volunteer 2 & 0 & 0 & 0 & 0 & 0 \\
Volunteer 3 & 68 & 68 & 68 & 68 & 68 \\
\hline With mask $(\mathrm{cm})$ & & & & & \\
\hline Volunteer 1 & 0 & 0 & 0 & 0 & 0 \\
Volunteer 2 & 0 & 0 & 0 & 0 & 0 \\
Volunteer 3 & 48 & 0 & 0 & 0 & 0 \\
\hline
\end{tabular}

The median (range) distances of microparticle scattering without the AP mask were 60 (60-60), 0, and 68 (68-68) in volunteers 1,2 , and 3 , respectively. The median (range) distances of microparticle scattering with the AP mask were 0,0 , and $0(0-48)$, respectively (Table 3$)$.

\section{Study 2}

\section{Patients characteristics}

A total of 34 lesions in 30 patients underwent EMR/ESD during the study period. Most of the patients were classified as Eastern Cooperative Oncology Group (ECOG) PS 0 and American Society of Anesthesiologists (ASA) Physical Status Classification II (Table 4). The most frequent comorbidity was gastric cancer (66.6\%).

Six esophageal EMRs, seven esophageal ESDs, and 21 gastric ESDs were performed. All lesions achieved en bloc resection. The mean ( \pm standard deviation $[\mathrm{SD}])$ procedural time was $42.4 \pm 5.1$ minutes (Table 5).

The mean dosage of propofol and pentazocine $( \pm S D)$ was $13.1 \pm 2.3 \mathrm{mg} / \mathrm{kg} / \mathrm{hr}$ and $16.8 \pm 0.9 \mathrm{mg}$, respectively. During sedation, mean $\mathrm{SpO} 2( \pm \mathrm{SD})$ was $96.3 \pm 0.7 \%$, and desaturation occurred in three patients (Table 6). Adverse events, such as aspiration pneumonia, were not observed. In all cases, droplets were identified inside the AP mask (Fig. 4).

Table 4. Patient's Characteristics $(n=30)$

\begin{tabular}{lc}
\hline Age (years) & $73.3 \pm 1.7$ \\
Gender, male/female & $25 / 5$ \\
Performance status (0/1) & $25 / 5$ \\
ASA classification (I/II/III) & $5 / 25 / 0$ \\
Height (cm) & $164.4 \pm 1.7$ \\
Body weight (kg) & $62.1 \pm 2.0$ \\
Comorbidity* & \\
Cardiovascular disease & $8(26.6)$ \\
Cerebrovascular disease & $1(3.3)$ \\
Esophageal cancer/Gastric cancer & $14 / 20(46.6 / 66.6)$ \\
Other malignant disease & $8(26.6)$ \\
Diabetes mellites & $6(20)$ \\
Hypertension & $14(46.6)$ \\
Respiratory functional disorder & $9(30)$ \\
\hline
\end{tabular}

Data are presented by mean \pm standard deviation or number (\%).

${ }^{*}$ With overlapping

ASA, American Society of Anesthesiologists. 
Table 5. Lesion Characteristics $(n=34)$

\begin{tabular}{lc}
\hline Esophageal cancer & 13 \\
EMR/ESD & $6 / 7$ \\
Gastric cancer & 21 \\
EMR/ESD & $0 / 21$ \\
Procedure time $(\mathrm{min})$ & $42.4 \pm 5.1$ \\
Size of the tumor $(\mathrm{mm})$ & $11.9 \pm 1.6$ \\
\hline
\end{tabular}

Data are presented by mean \pm standard deviation.

EMR, endoscopic mucosal resection; ESD, endoscopic submucosal dissection.

Table 6. Drugs and Dosages during Sedation $(n=30)$

\begin{tabular}{lc}
\hline Propofol & $30(100)$ \\
Dosage of drugs & \\
Propofol $(\mathrm{mg} / \mathrm{kg} / \mathrm{hr})$ & $13.1 \pm 2.3$ \\
Pentazocine $(\mathrm{mg})$ & $16.8 \pm 0.9$ \\
Minimum SpO2 (\%) & $96.3 \pm 0.7$ \\
Discontinuation cases & $0(0)$ \\
\hline
\end{tabular}

Data are presented by mean \pm standard deviation or number (\%).

\section{DISCUSSION}

This is the first study to evaluate the effectiveness of the AP mask through direct aerosol visualization during GI endoscopy simulation. In this study, microparticles were visualized during voluntary coughing. We compared the number of microparticles generated with and without the AP mask. Microparticles were dramatically reduced when the AP mask was worn. Although we could not distinguish the aerosol and floating material in the air in detail, most of the microparticles were thought to be aerosols in this study. Thus, the AP mask could prevent exposure to aerosols in the cleanroom. Furthermore, desaturation occurred in only three patients in this study, as reported in a previous study, ${ }^{14}$ and no adverse events such as aspiration pneumonia occurred. Therefore, the AP mask is clinically feasible and safe in clinical practice.

According to previous reports, exposure to SARS-CoV-2 can occur from face-to-face contact within 6 feet of a patient with symptomatic COVID-19. ${ }^{15}$ In our study, the aerosols spread at least 2 feet from the mouth when the volunteer coughed with a simulated gastroscope insertion. These results suggest that endoscopists are at risk of COVID-19 infection when not wearing a mask. Sagami et al. demonstrated that upper GI endoscopy is an aerosol-generating procedure by

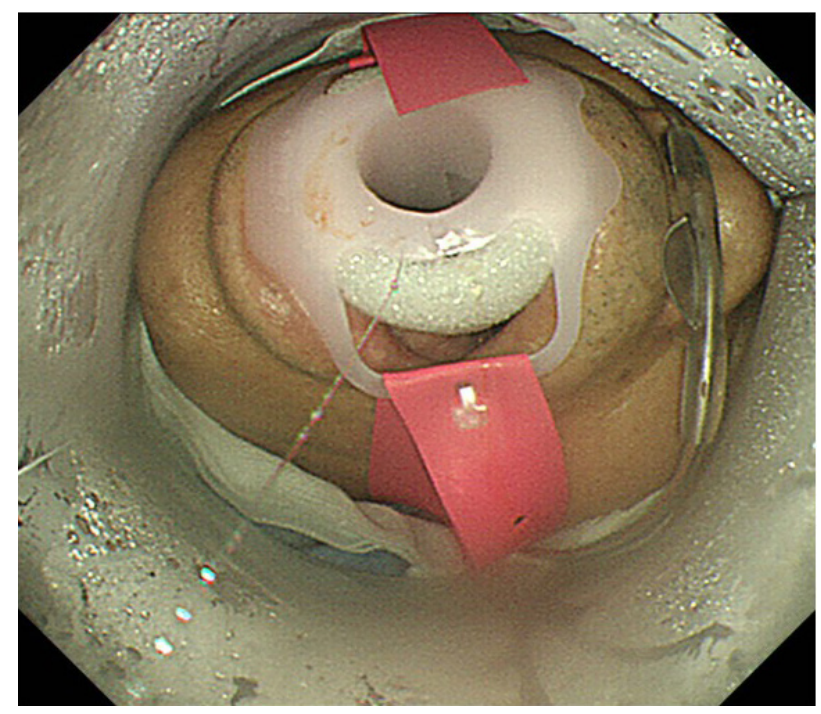

Fig. 4. Droplets were identified inside the aerosol-exposure protection mask during endoscopic procedures.

measuring 0.3-1.0 $\mu \mathrm{m}$ aerosols using an endoscopic shield. In this study, aerosol count was significantly higher during endoscopic procedures than in the control group. ${ }^{5}$ Our simulated experiment showed that microparticles were lower in the AP mask group than in the control group. Given these results, the AP mask could be used to cover patients' faces and prevent HCPs from coming into contact with the patient's aerosols during upper GI endoscopy in clinical settings.

Prevention of coronavirus infections in the endoscopy unit is essential for all endoscopic staff during the COVID-19 pandemic. As previously mentioned, PPE is useful for protecting HCPs from infection. However, previous reports have shown that the coronavirus remains viable in aerosols for up to 3 hours. ${ }^{16}$ Therefore, not only PPE but also aerosol prevention from the patient's standpoint is critical. Recently, several novel devices for protection against exposure to aerosols and droplets during GI endoscopy have been described. Sagami et al. developed an endoscopic shield with a plastic cube and demonstrated its efficacy. ${ }^{17}$ Although this device is fully enclosed, except for the hole for gastroscope insertion, which provides a great level of protection, it must be washed to re-use and is expensive (40 US dollars). Other studies have reported on the use of a disposal barrier with plastic sheets that cover the entire face and body during upper GI endoscopy. ${ }^{18,19} \mathrm{Sa}$ saki et al. made a glove-covered mouthpiece with a disposable glove. ${ }^{20}$ The patient bites the mouthpiece with the glove during the upper GI endoscopy, and aerosol particles are trapped by a rubber seal. Although this appears to be a simple and inexpensive solution, aerosol leakage from the side of the barrier or mouthpiece is concerning. In addition, these studies did not 
examine the visualization of aerosol particles. Several reports have used an anesthetic face mask to avoid aerosol spread of droplets. ${ }^{21,22}$ Marchese et al. reported that an anesthetic face mask (Flexicare; Flexicare Middle East and Africa, Amman, Jordan) can be used to prevent aerosol exposure during GI endoscopy, although no scientific data analysis is available.

Compared with previous reports, our single-use and inexpensive AP mask could entirely cover the patient's face. In addition, our study demonstrated the visualization of the aerosols and compared the number of microparticles with and without the AP mask, which were dramatically reduced in the former. In addition, no adverse events related to wearing the AP mask occurred during clinical use.

Our study had several limitations. First, this was a single-center simulation study with a small sample size. As this was an exploratory study, we did not set the sample size. In addition, protection from aerosol particles may not necessarily result in the prevention of COVID-19 infection. Second, we could not compare the control group (without the AP mask) in a real clinical setting. Therefore, further research with a larger sample size is warranted to confirm the results of this study.

In conclusion, the AP mask could assist in protecting HCPs against COVID-19 infection from aerosol-exposure during upper GI endoscopy.

\section{Conflicts of Interest}

Seiichiro Abe is currently serving as an associate editor in Clinical Endoscopy; however, he was not involved in the peer reviewer selection, evaluation, or decision process for this article. The authors have no potential conflicts of interest.

Funding

This study was supported by a research grant from Olympus Medical.

\section{Acknowledgments}

We would like to thank Dr. Lady Katherine Mejía Pérez (Gastroenterology, Cleveland Clinic Foundation) for her kind support of this article.

We also thank Shin Nippon Air Technologies, Co., Ltd. in Tokyo, Japan.

\section{Author Contributions}

Conceptualization: Mai Ego Makiguchi, Seiichiro Abe, Yutaka Okagawa

Data curation: MEM, SA

Formal analysis: MEM, SA

Project administration: MEM, SA, YO, Ryuta Okamoto

Supervision: Yutaka Saito

Writing-original draft: MEM, SA

Writing-review \& editing: MEM, SA, YO,RO, Satoru Nonaka, Haruhisa Suzuki, Shigetaka Yoshinaga, Ichiro oda, YS

\section{ORCID}

Mai Ego Makiguchi

Seiichiro Abe

Yutaka Okagawa https://orcid.org/0000-0002-3620-0907 https://orcid.org/0000-0002-2736-6921 https://orcid.org/0000-0002-1215-1901
Satoru Nonaka

Haruhisa Suzuki

Shigetaka Yoshinaga

Ichiro Oda

Ryuta Okamoto

Yutaka Saito https://orcid.org/0000-0002-0925-9137 https://orcid.org/0000-0003-2284-0943 https://orcid.org/0000-0002-2368-878X https://orcid.org/0000-0001-5252-4197 https://orcid.org/0000-0002-6879-624X https://orcid.org/0000-0003-2296-8373
Supplementary Materials

Video 1. Without the aerosol-exposure protection mask, the aerosol spread over 2 feet (https://doi.org/10.5946/ce.2021.178-IDEN.v001).

Video 2. With the aerosol-exposure protection mask, aerosol did not leak outside the mask (https://doi.org/10.5946/ce.2021.178-IDEN.v002).

\section{REFERENCES}

1. World Health Organization. Coronavirus disease (COVID-2019) situation reports - 5 January 2021 [Internet]. Geneva: WHO; c2021. [cited 2021 Oct 12]. Available from: https://www.who.int/publications/m/ item/weekly-epidemiological-update---5-January-2021.

2. Ortega R, Gonzalez M, Nozari A, Canelli R. Personal protective equipment and Covid-19. N Engl J Med 2020;382:e105.

3. The Lancet. COVID-19: protecting health-care workers. Lancet 2020;395:922.

4. Gralnek IM, Hassan C, Beilenhoff U, et al. ESGE and ESGENA position statement on gastrointestinal endoscopy and the COVID-19 pandemic. Endoscopy 2020;52:483-490.

5. Sagami R, Nishikiori H, Sato T, et al. Aerosols produced by upper gastrointestinal endoscopy: a quantitative evaluation. Am J Gastroenterol 2021;116:202-205.

6. Bhandari P, Subramaniam S, Bourke MJ, et al. Recovery of endoscopy services in the era of COVID-19: recommendations from an international Delphi consensus. Gut 2020;69:1915-1924.

7. Irisawa A, Furuta $\mathrm{T}$, Matsumoto $\mathrm{T}$, et al. Gastrointestinal endoscopy in the era of the acute pandemic of coronavirus disease 2019: recommendations by Japan Gastroenterological Endoscopy Society (Issued on April 9th, 2020). Dig Endosc 2020;32:648-650.

8. Repici A, Maselli R, Colombo M, et al. Coronavirus (COVID-19) outbreak: what the department of endoscopy should know. Gastrointest Endosc 2020;92:192-197.

9. Chiu PWY, Ng SC, Inoue H, et al. Practice of endoscopy during COVID-19 pandemic: position statements of the Asian Pacific Society for Digestive Endoscopy (APSDE-COVID statements). Gut 2020;69:991-996.

10. Hennessy B, Vicari J, Bernstein B, et al. Guidance for resuming GI endoscopy and practice operations after the COVID-19 pandemic. Gastrointest Endosc 2020;92:743-747.e1.

11. Sawano T, Kotera Y, Ozaki A, et al. Underestimation of COVID-19 cases in Japan: an analysis of RT-PCR testing for COVID-19 among 47 prefectures in Japan. QJM 2020;113:551-555.

12. Kawashima K, Abe S, Koga M, et al. Optimal selection of endoscopic resection in patients with esophageal squamous cell carcinoma: endoscopic mucosal resection versus endoscopic submucosal dissection according to lesion size. Dis Esophagus 2021;34:doaa096.

13. Mori G, Nonaka S, Oda I, et al. Novel strategy of endoscopic submucosal dissection using an insulation-tipped knife for early gastric cancer: nearside approach method. Endosc Int Open 2015;3:E425-E431.

14. Nonaka S, Kawaguchi Y, Oda I, et al. Safety and effectiveness of propofol-based monitored anesthesia care without intubation during endoscopic submucosal dissection for early gastric and esophageal cancers. Dig Endosc 2015;27:665-673.

15. Klompas M, Morris CA, Shenoy ES. Universal masking in the Covid-19 era. N Engl J Med 2020;383:e9. 
16. van Doremalen N, Bushmaker T, Morris DH, et al. Aerosol and surface stability of SARS-CoV-2 as compared with SARS-CoV-1. N Engl J Med 2020;382:1564-1567.

17. Sagami R, Nishikiori H, Sato T, Murakami K. Endoscopic shield: barrier enclosure during the endoscopy to prevent aerosol droplets during the COVID-19 pandemic. VideoGIE 2020;5:445-448.

18. Leow VM, Mohamad IS, Subramaniam M. Use of aerosol protective barrier in a patient with impending cholangitis and unknown COVID-19 status undergoing emergency ERCP during COVID-19 pandemic. BMJ Case Rep 2020;13:e236918.

19. Sabbagh L, Huertas M, Preciado J, Sabbagh D. New protection barrier for endoscopic procedures in the era of pandemic COVID-19. Video-
GIE 2020;5:614-617.

20. Sasaki S, Nishikawa J, Sakaida I. Use of a glove-covered mouthpiece during upper endoscopy to prevent COVID-19 transmission. Clin Endosc 2021;54:289-290.

21. Marchese M, Capannolo A, Lombardi L, Di Carlo M, Marinangeli F, Fusco P. Use of a modified ventilation mask to avoid aerosolizing spread of droplets for short endoscopic procedures during coronavirus COVID-19 outbreak. Gastrointest Endosc 2020;92:439-440.

22. Tian Q, Yan X, Shi R, et al. Endoscopic mask innovation and protective measures changes during the coronavirus disease-2019 pandemic: experience from a Chinese hepato-biliary-pancreatic unit. Dig Endosc 2020;32:1105-1110. 